\title{
ISCEV extended protocol for the dark-adapted red flash ERG
}

\author{
Dorothy A. Thompson • Kaoru Fujinami • Ido Perlman • Ruth Hamilton • \\ Anthony G. Robson
}

Received: 4 May 2018/Accepted: 8 May 2018/Published online: 22 June 2018

(C) The Author(s) 2018

\begin{abstract}
The International Society for Clinical Electrophysiology of Vision (ISCEV) standard for full-field electroretinography (ERG) describes a minimum procedure, but encourages more extensive testing. This ISCEV extended protocol describes an extension to the ERG standard, namely the darkadapted (DA) red flash ERG. The DA red flash ERG can be incorporated conveniently within the ISCEV standard ERG protocol after a minimum of 20-min DA and recorded after the DA 0.01 ERG to a flash strength
\end{abstract}

D. A. Thompson ( $₫)$

The Tony Kriss Visual Electrophysiology Unit, Clinical and Academic Department of Ophthalmology, Great Ormond Street Hospital for Children NHS Trust, London, UK

e-mail: dorothy.thompson@gosh.nhs.uk

\section{A. Thompson}

UCL Great Ormond Street Institute for Child Health, London, UK

\section{K. Fujinami}

Laboratory of Visual Physiology, Division for Vision Research, National Institute of Sensory Organs, National Hospital Organization, Tokyo Medical Centre, Tokyo, Japan

\section{K. Fujinami}

Department of Ophthalmology, Keio University School of Medicine, Tokyo, Japan

K. Fujinami - A. G. Robson

UCL Institute of Ophthalmology, London, UK of 0.3 phot $\mathrm{cd} \mathrm{s} \mathrm{m}^{-2}$, eliciting a waveform with two positive peaks in healthy individuals. The first positive component is the cone-mediated $\mathrm{x}$-wave with a peak at $30-50 \mathrm{~ms}$; the second is a rod-mediated b-wave with a peak time of approximately $100 \mathrm{~ms}$. Shorter DA times may be desirable to shorten the recording time or to alter the prominence of the early cone-mediated $\mathrm{x}$-wave relative to the rod-mediated b-wave. The DA red flash ERG is used to aid the diagnosis of achromatopsia (rod monochromacy), cone dystrophy

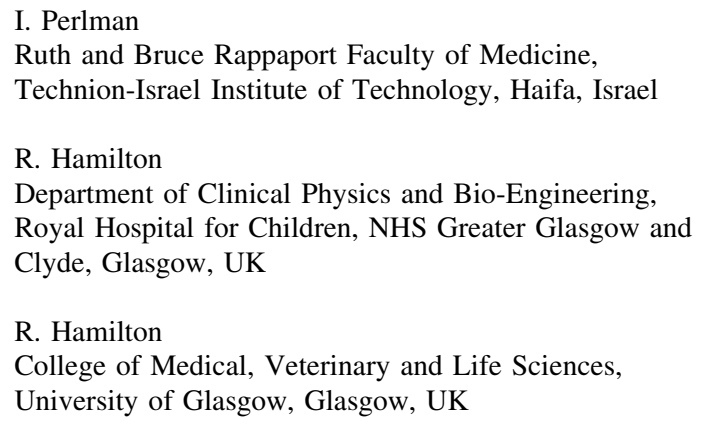

A. G. Robson

Electrophysiology Department, Moorfields Eye Hospital, London, UK 
and other forms of cone system dysfunction, including "Bradyopsia" (RGS9/R9AP-retinopathy), when the DA red flash ERG $\mathrm{x}$-wave is preserved in the absence of ISCEV standard LA ERGs. The DA red flash ERG can also help determine the origin of residual DA ERGs in cases of severe rod dysfunction, for example in disorders such as vitamin A deficiency, fundus albipunctatus (RDH5-retinopathy), Oguchi disease (SAG- or GRK1-retinopathy) and some rod-cone dystrophies. To shorter DA periods, the x-wave may be elicited without the following rod b-wave, shown to be helpful in abbreviated protocols for children.

Keywords Clinical standards - Electroretinogram (ERG) · Full-field ERG · International Society of Clinical Electrophysiology of Vision (ISCEV) · Darkadapted (DA) · Red flash ERG · Retinal dystrophy

\section{Introduction}

The International Society for Clinical Electrophysiology of Vision (ISCEV) standard for full-field electroretinography (ERG) describes a minimum set of tests, but encourages the use of additional ERG protocols for clinical ERG testing [1]. This extended protocol describes the dark-adapted (DA) red ERG, as a specialized procedure which is well established and broadly accepted by experts in the field. The protocol was prepared by the authors in accordance with ISCEV procedures (http://www.iscev.org/standards/ index.html) and was approved by the ISCEV Board of Directors on March 25, 2018.

\section{Scope and applications}

The ISCEV ERG standard [1] describes a minimum protocol to test generalized rod and cone system function in the outer and inner retina. The DA red flash ERG can be used to distinguish the function of DA rod and cone systems and can help determine the origins of abnormal standard flash ERGs, which may be important for accurate characterization of retinal function and to establish some diagnoses. This extended protocol describes parameters for the dark-adapted (DA) red flash ERG that may be added to the ISCEV standard ERG protocol.
The normal cone system contributes to the full-field ERG under DA as well as light-adapted (LA) conditions. This occurs in DA ERGs evoked by flash strengths greater than $0.1 \mathrm{~cd} \mathrm{~s} \mathrm{~m}^{-2}$ [2], including the ISCEV standard DA 3 ("combined rod cone") and DA 10 ("strong flash") ERGs. Early investigations revealed the contribution of DA cones in the ERG waveform by using colored flashes that exploited differences in the spectral sensitivities of rods and cones [3-5]. These studies showed that the DA ERG waveform to a red flash has two distinct positive peaks. The first, named the $\mathrm{x}$-wave, occurred within 30-50 ms and was attributed to DA cone activity. The $\mathrm{x}$-wave was followed by a rod-mediated $\mathrm{b}$-wave [3]. The $\mathrm{x}$-wave is larger than the b-wave during the early stages of dark adaptation when the rod system threshold is high. As dark adaptation proceeds, the xand $b$-wave amplitudes become similar and finally the b-wave exceeds the $\mathrm{x}$-wave [6].

The DA red flash ERG has several clinical applications, and the circumstances and diagnoses that may benefit from testing are outlined below:

(a) The DA red flashes are usually well tolerated by patients of all ages, and the test is therefore useful if photophobia or photo-aversion confounds the recording of standard LA ERGs. This can occur in the presence of cone dysfunction, but also, for example, in the presence of media opacity or strong Bell's phenomenon.

(b) In cases of generalized cone system dysfunction such as rod- and S-cone monochromacy and cone dystrophy, the DA red flash ERG x-wave may be undetectable, markedly attenuated and/ or delayed [7-10].

(c) In cases of generalized retinal dysfunction, the relative involvement of the DA red flash ERG $\mathrm{X}$-wave and $\mathrm{b}$-wave may suggest predominant dysfunction of cone or rod systems, not always obvious by comparing standard DA and LA ERGs.

(d) In cases of severe or selective rod dysfunction, the DA red flash ERG can help determine the causes and origins of abnormal or residual DA bright flash ERGs. This occurs, for example, in vitamin A deficiency [11], fundus albipunctatus (RDH5-retinopathy) [12] and Oguchi disease (SAG- or GRK1-retinopathy) [13] and in some cases of rod-cone dystrophy including early 
stages of Bothnia dystrophy (RLBP1-retinopathy). In these disorders, the DA 3 and DA 10 ERGs have reduced a-waves indicating rod photoreceptor dysfunction, but there may also be reduction in the b:a ratio and shortening of b-wave peak time in the absence of a rod system contribution. The reduced $b$ :a ratio may arise from strong stimulation of the relatively preserved DA cone system, analogous to the photopic hill phenomenon, and produces a b-wave which resembles the DA red flash ERG X-wave.

(e) "Bradyopsia" (RGS9- and R9AP-retinopathy). The DA red flash ERG is normal, but LA conemediated ERGs are extinguished by repetitive flashes [14, 15]. The combination of a preserved DA red flash ERG $x$-wave and undetectable or severely abnormal standard LA ERGs is pathognomonic for the disorder.

(f) The red flash ERG has been used to detect color vision deficiencies and has been reported to be absent $[9,16]$ or subnormal [10] in protanopia. The implication is that around $1 / 100$ males would have an absent red flash ERG although this has not been established for an ISCEV DA red flash ERG extended protocol.

\section{Patient population}

Patients of all ages, referred for investigation of possible retinal dysfunction, retinal dystrophy, generalized cone or rod system dysfunction or patients with photophobia may benefit from the DA red flash ERG.

\section{Technical issues}

The DA red flash ERG will follow the specifications of the current ISCEV standard full-field ERG and for most applications may be embedded within the standard protocol [1].

Additional considerations include the following:

(a) The spectral characteristics of the red flash. Both peak wavelength and bandwidth may affect the DA red flash ERG. Physical filters, e.g., Kodak Wratten filters 26 (dominant wavelength $619 \mathrm{~nm}$ ) or 29 (dominant wavelength $630 \mathrm{~nm}$ ), were used in many older studies, but have been largely superseded by LEDs, e.g. peak wavelengths 635 or $655 \mathrm{~nm}$, and choice may be equipment dependent. It is noted that peak wavelengths shorter than $620 \mathrm{~nm}$ may be perceived as orange, and wavelengths longer than $630 \mathrm{~nm}$ provide slightly better separation of $\mathrm{x}$-wave and b-wave, and that for wavelengths longer than $650 \mathrm{~nm}$ waveforms have been reported with a third positive wave, later than the rod b-wave [6].

(b) The units of flash strength. The relative (effective) strength of a colored flash depends upon the adaptation and hence spectral sensitivity of the eye. Absolute measures are radiant energy, but for uniformity of clinical use and consistency with other flash stimuli, photometric units defined in phot $\mathrm{cd} \mathrm{s} \mathrm{m}^{-2}$ are recommended.

(c) Duration of dark adaptation. The choice of dark adaptation duration and flash strength depends upon one of three aims (Fig. 1):

1. To isolate the cone-mediated $\mathrm{x}$-wave (peak time 30-50 ms): short dark-adaptation of around $5 \mathrm{~min}$ reveals the $\mathrm{x}$-wave before it is masked by full development of the later rod-mediated b-wave $[6,8,17]$.

2. To separate the $\mathrm{x}$ - and $\mathrm{b}$-wave peak times: if an ISCEV standard period of at least 20-min dark adaptation is used, weaker red flash strengths of around $0.03-0.3 \mathrm{~cd} \mathrm{~s} \mathrm{~m}^{-2}$ allow maximum separation in time of the cone- and rod-mediated components.

3. To match the amplitudes of the DA red flash ERG b-wave with the ISCEV standard DA 0.01 ERG (rod ERG) b-wave, different red flash strengths may be needed depending upon the patient's age, as the DA red flash rod b-wave diminishes with age, relative to the DA red flash $\mathrm{x}$-wave [18].

(d) Frequency of red flash presentation. The interstimulus interval will influence the light adaptation of the retina and shape of the DA red flash ERG waveform [19]. The ISCEV standard for the DA 0.01 ERG is less than or equal to 1 flash every $2 \mathrm{~s}$, and a similar frequency may be 


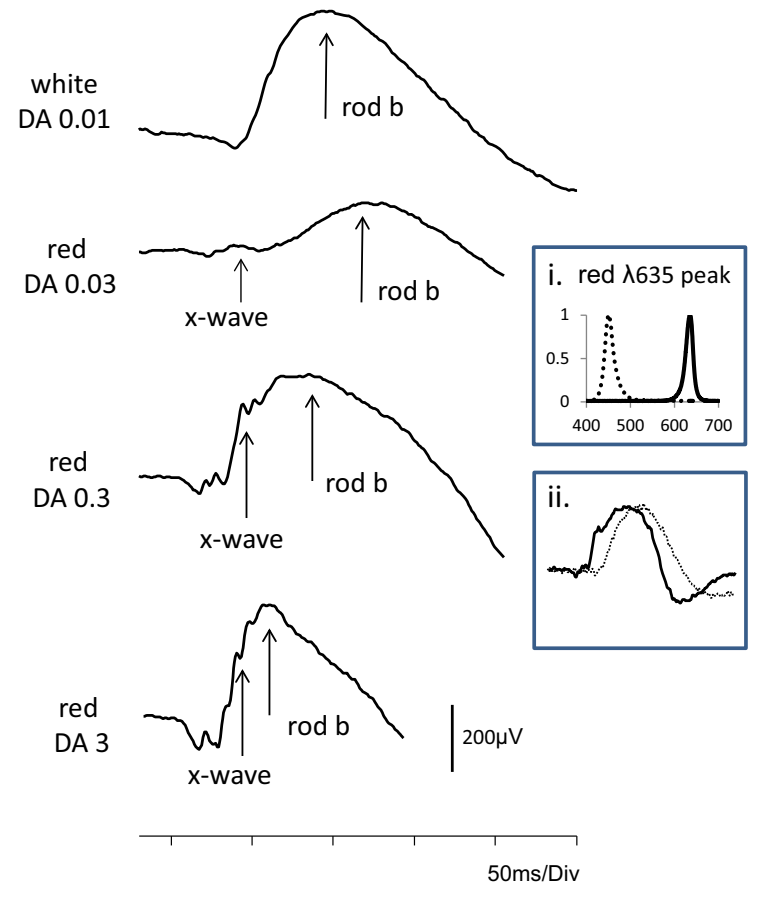

Fig. 1 An ISCEV standard DA 0.01 ERG is shown at the top of the figure to compare the waveform of the rod driven $b$-wave with that of DA red flash ERGs produced by three different flash strengths of wavelength $635 \mathrm{~nm}$ after 20-min dark adaptation. Note the separation in peak time of the $\mathrm{x}$-wave and $\mathrm{b}$-wave to DA $0.03 \mathrm{~cd} \mathrm{~s} \mathrm{~m}^{-2}$ (dim) flashes, the enlargement of the $\mathrm{x}$-wave to DA $0.3 \mathrm{~cd} \mathrm{~s} \mathrm{~m}^{-2}$ and the merging of $\mathrm{x}$ - and b-waves at DA 3 in a control subject. Insert $i$ shows the spectral characteristics of the red (solid line) and blue (dotted line) LEDs in the Ganzfeld. Insert ii shows the DA red flash ERG to $0.3 \mathrm{~cd} \mathrm{~s} \mathrm{~m}^{-2}$ in a second subject compared with a DA blue flash ERG of "scotopically matched" b-wave amplitude, in this case DA blue $0.0003 \mathrm{~cd} \mathrm{~s} \mathrm{~m}^{-2}$ (the red flash response may also be "matched" to a DA dim white flash ERG). DA red ERG is shown as a solid black lines and DA blue flash ERG as a dotted line

appropriate for flash strengths that elicit responses of similar amplitude to the DA 0.01 ERG.

\section{Calibration}

Calibration is in accordance with the ISCEV ERG standard [1]. A spectral photometer is required to determine the spectral characteristics of the red flash. Stimulators may use different combinations of LEDs for different flash strengths, so equal spectral characteristics should not be assumed.

\section{Protocol specification}

Patient preparation follows that of the current ISCEV ERG standard [1] and the DA red flash ERG may be embedded within the standard ERG protocol. Additional specifications are listed below:

(a) Stimulus wavelength For routine diagnostic applications, LEDs with a peak wavelength of between $635 \mathrm{~nm}$ (Fig. 1) and $650 \mathrm{~nm}$ (Fig. 2) are suggested to allow separation of $\mathrm{x}$ - and b-waves. If Xenon flashes and filters are used, a dominant wavelength of $619 \mathrm{~nm}$ (e.g., Wratten 26) or $630 \mathrm{~nm}$ (e.g., Wratten 29) may be used. The peak wavelength and bandwidth at halfheight of the stimulus and method of generation (optical filter or LED) should be stated.

(b) Flash strength The minimum dark-adapted red flash protocol includes a red flash strength of $0.3 \mathrm{~cd} \mathrm{~s} \mathrm{~m}^{-2}$. This does not preclude the recording of additional red flash ERGs (ranging around $0.3 \mathrm{~cd} \mathrm{~s} \mathrm{~m}^{-2}$; see Fig. 2), but care should be taken to avoid light-adapting the retina with higher flash strengths, and it may be necessary to increase the inter-stimulus interval. If an additional red flash is defined as that required to elicit a DA red flash ERG rod system b-wave of equal or similar amplitude to the DA

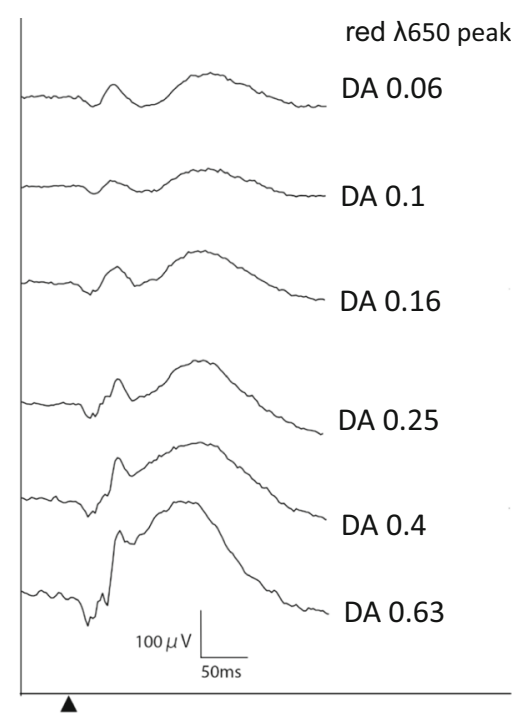

Fig. 2 The gradual change in waveform of DA red flash ERG to a range of flash strengths weaker or stronger than $0.3 \mathrm{~cd} \mathrm{~s} \mathrm{~m}^{-2}$, recorded after $20 \mathrm{~min}$ DA with a $650 \mathrm{~nm}$ red flash 
Table 1 A summary of published DA red flash ERG parameters

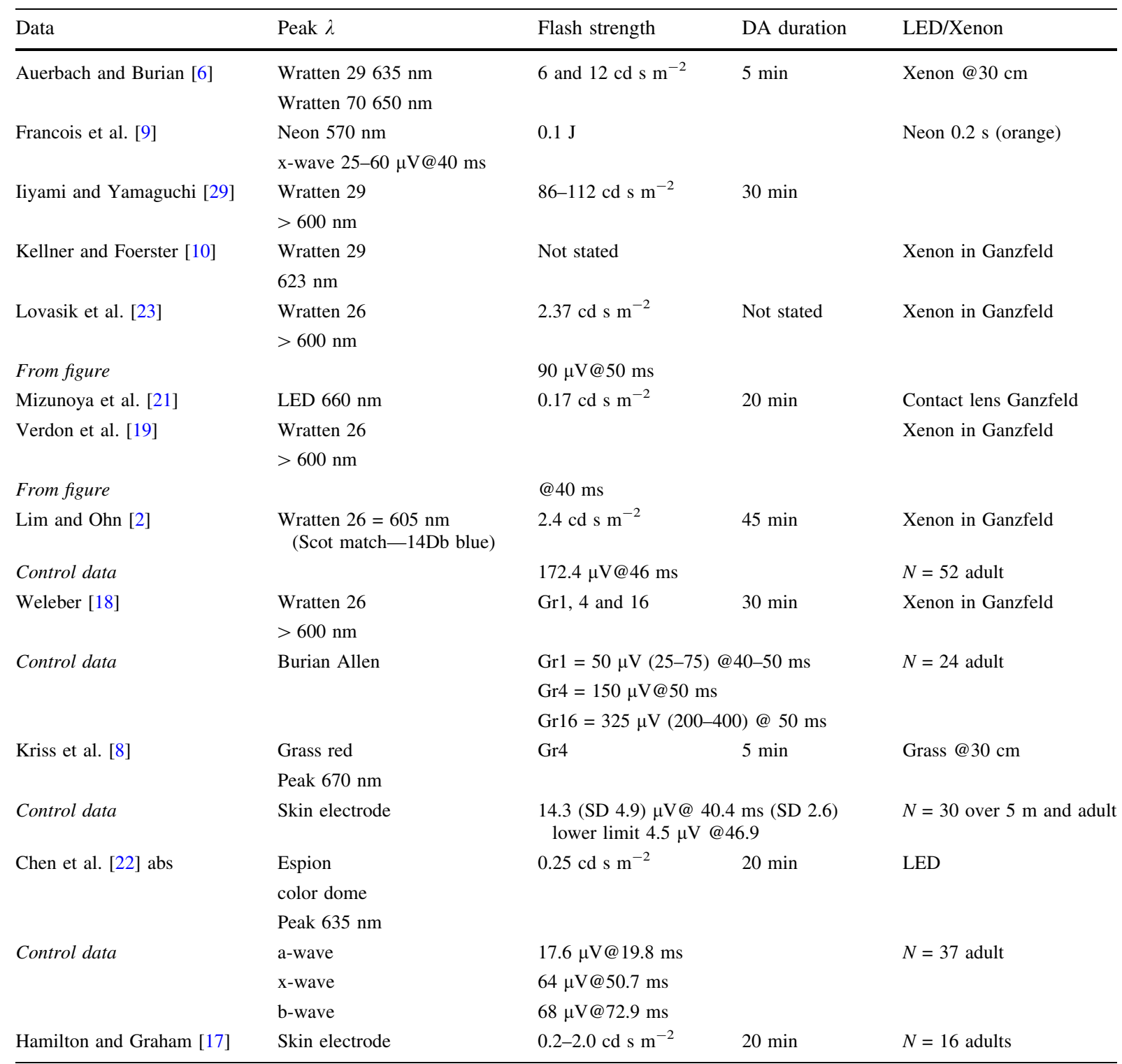

0.01 ERG or to a DA blue flash ERG, this should be acknowledged and the corresponding flash stimuli stated in phot $\mathrm{cd} \mathrm{s} \mathrm{m}^{-2}$.

(c) Duration of dark adaptation The duration of dark adaptation required to record a darkadapted red flash depends on the aims. The DA red flash ERG can be incorporated conveniently within the ISCEV standard ERG protocol after a minimum of 20-min DA, after the standard DA 0.01 ERG. Shorter DA times may be desirable to shorten the recording time or specifically to reduce the rod system b-wave and increase the prominence of the cone system $\mathrm{x}$-wave (see above). Mesopic cone-rod interactions associated with shorter DA may increase the variability of the DA red ERG b-wave amplitude.

(d) Frequency of red flash presentation A flash rate of $\leq 0.5 / \mathrm{s}$ is recommended (inter-stimulus interval $\geq 2 \mathrm{~s}$ ). This conforms to the current 
ISCEV standard for the DA 0.01 ERG. Longer inter-stimulus intervals may be needed for stronger red flashes.

\section{Response evaluation}

Examples are shown in Figs. 1 and 2 of the DA red flash ERG waveforms produced by different flash strengths delivered using an LED (peak wavelength 635 or $650 \mathrm{~nm}$ ). For routine testing, it is recommended that the $\mathrm{x}$-wave and $\mathrm{b}$-wave peak times and amplitudes are measured and reported. Peak times are measured from the flash (mid-point) and amplitudes from the baseline or a-wave (earliest negative trough) if present.

\section{Reporting}

Reporting the DA red flash should follow the recommendations of the ISCEV ERG protocol. The flash stimulus characteristics (LED or filter), peak wavelength or filter specification (e.g., Wratten 26 or 29) should be stated. The flash strength should be stated. Unless already embedded within the ISCEV standard ERG protocol, pupil size and duration of dark adaption should be stated. The amplitude of the a-wave, $x$-wave and $b$-wave and their respective time to peaks may be reported along with age-appropriate laboratory reference data. It is acknowledged that in studies involving ISCEV standard ERGs it may be sufficiently informative to describe the relative reduction or preservation of $\mathrm{x}$-wave and/or b-waves relative to each other and reference ranges.

Acknowledgements We would like to thank the members of ISCEV and BriSCEV and in particular Donnell Creel, Chris Hogg, Michael F. Marmor, Daphne McCulloch, Yves Sauvé and Mineo Kondo for their valuable discussion during the consultation period.

\section{Compliance with ethical standards}

Conflict of interest The authors have no conflicts of interest.

Informed consent As this article does not contain any studies with human participants performed directly by any of the authors, the concept of informed consent is not applicable.
Statement of human rights The study involved no research on Human participants and consent is not applicable.

Statement on the welfare of animals The study involved no research on animals.

Open Access This article is distributed under the terms of the Creative Commons Attribution 4.0 International License (http:// creativecommons.org/licenses/by/4.0/), which permits unrestricted use, distribution, and reproduction in any medium, provided you give appropriate credit to the original author(s) and the source, provide a link to the Creative Commons license, and indicate if changes were made.

\section{Appendix: Justification for the protocol details}

We followed the Preferred Reporting Items for Systematic Reviews and Meta-Analyses (PRISMA) [20] when writing this report. The search strategy aimed to identify reports of scotopic red flash ERGs in order to extract stimulus parameters of wavelength, flash strength, stimulus duration, temporal frequency, dark adaptation period and amass evidence of its clinical application and range of response expected in normal and clinical cases.

A systematic literature review was performed to find publications that reported the scotopic red flash ERG initially from the period January 1942 to April 10, 2017 using MEDLINE, EMBASE and Cochrane reviews. Exclusion criteria were animal studies and absence of any stimulus specification. In summary, red flash strengths used, included 0.05, 0.1 [7], 0.17 [21], $0.25,0.5,0.75,1 \& 1.5$ [22], 2.37 [23], 2.4 [2], 1.5 and 2.5 [24], and $8 \mathrm{~cd} \mathrm{~s} \mathrm{~m}^{-2}$ [25] (personal communication Sauve 2017) after 20 min DA. A range of Grass stroboscope flash settings have been stated as 1, 4, 8 and 16 e.g. gr4 white PS22 $3.7 \times 10^{5}$ candles [26] or gr $4+$ Wratten 26 filter $=0.02 \log \mu \mathrm{J} / \mathrm{cm}^{2}$-steradian [18]. Some studies report flash strengths "such that in a normal subject the amplitude of the rod component to the red flash ERG is equivalent to that of the rodspecific response to a dim white flash (dark-adapted $0.01 \mathrm{~cd} \mathrm{~s} \mathrm{~m}^{-2}$ )" $[11,12,15,27]$ or report that the red flash luminance empirically be set to elicit a b-wave of $\sim 200 \mu \mathrm{V}$ [28]. The DA red flash ERG $\mathrm{x}$-wave is reported to be stable between 10-70 years of age whilst the rod-driven b-wave diminishes with increasing age, [18]. To match b-wave amplitudes in the DA 0.01 and DA red flash ERGs it may be necessary to increase the red flash strength in older individuals. The 
main published parameters are summarized in Table 1.

\section{References}

1. McCulloch DL, Marmor MF, Brigell MG, Hamilton R, Holder GE, Tzekov R et al (2015) ISCEV Standard for fullfield clinical electroretinography (2015 update). Doc Ophthalmol 130(1):1-12. https://doi.org/10.1007/s10633-0149473-7

2. Lim SH, Ohn YH (2005) Study of blue and red flash in darkadapted electroretinogram. Korean J Ophthalmol 19(2):106-111

3. Motokawa K, Mita T (1942) Über eine einfachere Untersuchungsmethode und Eigenschaften der Aktionsströme der Netzhaut des Menschen. Tohoku J Exp Med 42:114-133. https://doi.org/10.1620/tjem.42.114

4. Adrian ED (1945) The electric response of the human eye. J Physiol 104(1):84-104

5. Adrian ED (1946) The rod and cone components in the electrical response of the human eye. J Physiol 104:84-104. https://doi.org/10.1113/jphysiol.1945.sp004109

6. Auerbach E, Burian HM (1955) Studies on the photopicscotopic relationships in the human electroretinogram. Am J Ophthalmol 40(5):42-60

7. Miyake Y (2006) Electrodiagnosis of retinal disease. Springer, Japan, p 16-19

8. Kriss A, Jeffrey B, Taylor D (1992) The electroretinogram in infants and young children. J Clin Neurophysiol 9(3):373-393

9. Francois J, Verriest G, De Rouck A (1956) Pathology of the $\mathrm{X}$-wave of the human electroretinogram. I. Red-blindness and other congenital functional abnormalities. Br J Ophthalmol 40(7):439-443

10. Kellner U, Foerster MH (1992) Color electroretinography. A method for separation of dysfunctions of cones. Doc Ophthalmol Adv Ophthalmol 80(1):13-23

11. McBain VA, Egan CA, Pieris SJ, Supramaniam G, Webster AR, Bird AC, Holder GE (2007) Functional observations in vitamin A deficiency: diagnosis and time course of recovery. Eye 21:367-376. https://doi.org/10.1038/sj.eye. 6702212

12. Sergouniotis PI, Sohn EH, Li Z, McBain VA, Wright GA, Moore AT et al (2011) Phenotypic variability in RDH5 retinopathy (fundus albipunctatus). Ophthalmology 118(8):1661-1670. https://doi.org/10.1016/j.ophtha.2010. 12.031

13. Sergouniotis PI, Davidson AE, Sehmi K, Webster AR, Robson AG, Moore AT (2011) Mizuo-Nakamuraphenomenon in Oguchi disease due to a homozygous nonsense mutation in the SAG gene. Eye(Lond) 25(8):1098-1101. https://doi.org/10.1038/eye.2011.88

14. Michaelides M, Li Z, Rana NA, Richardson EC, Hykin PG, Moore AT, Holder GE, Webster AR (2010) Novel mutations and electrophysiologic findings in RGS9- and R9AP- associated retinal dysfunction (Bradyopsia). Ophthalmology $117(1): 120-127$

15. Cheng JYC, Luu CD, Yong VHK, Mathur R, Aung T, Vithana EN (2007) Bradyopsia in an Asian man. Arch Ophthalmol 125:1138-1140. https://doi.org/10.1001/ archopht.125.8.1138

16. Von Schubert G, Bornschein H (1952) Beitrag zur Analyse des menschlichen Elektroretinogramms. Ophthalmologica 123(6):396-412

17. Hamilton R, Graham K (2018) Dark-adapted red flash ERGs in healthy adults. Doc Ophthalmol. https://doi.org/10.1007/ s10633-018-9642-1

18. Weleber RG (1981) The effect of age on human cone and rod ganzfeld electroretinograms. Invest Ophthalmol Vis Sci 20(3):392-399

19. Verdon WA, Schneck ME, Haegerstrom-Portnoy G (2003) A comparison of three techniques to estimate the human dark-adapted cone electroretinogram. Vis Res 43(19):2089-2099

20. Moher D, Shamseer L, Clarke M, Ghersi D, Liberati A, Petticrew M et al (2015) Preferred reporting items for systematic review and meta-analysis protocols (PRISMA-P) 2015 statement. Syst Rev 4(1):1. https://doi.org/10.1186/ 2046-4053-4-1

21. Mizunoya S, Kuniyoshi K, Arai M, Tahara K, Hirose T (2001) Electroretinogram contact lens electrode with tricolor light-emitting diode. Acta Ophthalmol Scand 79(5):497-500

22. Chen L, Png R, Mathur R, Chia A (2015) Scotopic red ERG findings. In: 53rd symposium of International Society for Clinical Electrophysiology of Vision (ISCEV). Springer, Heidelberg, p 31

23. Lovasik JV, Kothe AC, Kergoat H (1992) Improving the diagnostic power of electroretinography by transient alteration of the ocular perfusion pressure. Optom Vis Sci 69(2):85-94

24. Chia A, Png R, Mathur R (eds) (2014) Scotopic red response: rod and cone components. In: International Society for Clinical Electrophysiology of Vision Symposium. Doc Ophthalmol, Boston

25. Freund PR, Watson J, Gilmour GS, Gaillard F, Sauve Y (2011) Differential changes in retina function with normal aging in humans. Doc Ophthalmol Adv Ophthalmol 122(3):177-190. https://doi.org/10.1007/s10633-011-92732

26. Kriss A, Russell-Eggitt I. Electrophysiological assessment of visual pathway function in infants. Eye (Lond). 1992;6(Pt 2):145-153. https://doi.org/10.1038/eye.1992.30

27. Vincent A, Robson AG, Holder GE (2013) Pathognomonic (diagnostic) ERGs. A review and update. Retina 33(1):5-12. https://doi.org/10.1097/IAE. 0b013e31827e2306

28. Creel DJ (ed) (2013) Scotopic dim blue and red ERG stimuli. Doc Ophthalmol

29. Iijima H, Yamaguchi S (1990) Adaptational changes in cone electroretinograms in man. Nippon Ganka Gakkai Zasshi Acta Soc Ophthalmol Jpn 94(11):987-992 